\title{
Establishment of a National Program for Quality Control of Nuclear Medicine Instrumentation
}

\author{
Marco A. Coca Perez ${ }^{1}$, Leonel A. Torres Aroche ${ }^{1}$, Gladys López Bejerano², Roberto Fraxedas Mayor ${ }^{3}$, \\ Consuelo Varela Corona ${ }^{4}$, and Adlin López ${ }^{5}$ \\ ${ }^{I}$ Center for Clinical Research, Habana, Cuba; ${ }^{2}$ Center for the Protection and Hygiene of Radiation, Habana, Cuba; \\ ${ }^{3}$ Nephrology Institute, Habana, Cuba; ${ }^{4}$ National Control Center for Medical Devices, Habana, Cuba; and \\ ${ }^{5}$ Hermanos Amejeiras Hospital, Habana, Cuba
}

\begin{abstract}
Monitoring the quality of instrumentation used in nuclear medicine is mandatory to guarantee the clinical efficacy of medical practice. A national program for the quality control of nuclear medicine instruments was established in Cuba and was certified and approved by the regulatory authorities. The program, which establishes official regulations and audit services, sets up educational activities, distributes technical documentation, and maintains a national phantom bank, constitutes a valuable and useful tool to guarantee the quality of nuclear medicine instrumentation.
\end{abstract}

Key Words: instrumentation; quality assurance; quality control; SPECT; gamma camera

J Nucl Med Technol 2008; 36:203-206

DOI: $10.2967 /$ jnmt.108.053504

$\mathbf{T}$ he correct functioning of instruments is essential for a successful nuclear medicine practice. Quality control programs for nuclear medicine equipment guarantee that clinical studies are of the highest quality, combining accuracy, precision, and rigor with practical criteria. Cuba has 10 gamma-cameras and SPECT systems installed in 9 nuclear medicine departments. This equipment has historically been evaluated and monitored according to institutional quality control programs based on international standards (e.g., protocols from organizations such as the National Electrical Manufacturers Association (NEMA), the International Atomic Energy Agency (IAEA), and the American Association of Physicists in Medicine), taking into account the local availability of clinical and standard phantoms, radioactive sources, and other resources.

A national program was organized and established to coordinate activities related to the quality control of nuclear medicine instrumentation. The program was the result of a 3-y multiinstitutional project. A group of experienced

Received Apr. 15, 2008; revision accepted Aug. 20, 2008.

For correspondence or reprints contact: Marco A. Coca Perez, Center for Clinical Research, Calle 34 No. 4501 e/ 45 y 47, Rpto Kohly, Playa, C. Habana, Cuba.

E-mail: mcoca@infomed.sld.cu

COPYRIGHT @ 2008 by the Society of Nuclear Medicine, Inc. medical physicists working in the nuclear medicine fieldfrom research centers, hospitals, and other local institutionsparticipated in this work.

The proposed quality control program for nuclear medicine instrumentation included the following aspects: implementation of national protocols and regulations according to local conditions and resources, education and training, organization and implementation of a phantom bank, establishment and registration of an annual audit service, and evaluation of the current state of nuclear medicine instruments.

As a first step, a national survey was performed to collect data about the features of the instruments, the availability of documentation, accessories and phantoms for quality control procedures, and the qualifications of the personnel responsible for quality control of all nuclear medicine services.

\section{NATIONAL PROTOCOLS}

A document entitled "National Protocols for Quality Control of Nuclear Medicine Instruments" was created. The objective was to develop national guidelines for a set of standardized and uniform quality control procedures, taking into account the technical features of the local instrumentation and the availability of local resources such as phantoms. These protocols are based on international publications such as those of the IAEA and NEMA, as well as the expertise of the working group (1-5). The document includes 6 chapters related to the quality control of dose calibrators, directional detectors and well counters, planar gammacameras, SPECT systems, whole-body systems, and interface systems. Each chapter is divided into 3 parts: a short introduction, basic and physical principles of work, and a detailed description of the quality control test including the objectives, materials necessary, technical procedures, analysis of data, and acceptance limits for the results. The document was evaluated and approved by the Cuban regulatory authorities (Centro de Control Estatal de Equipos Médicos [CCEEM]) as the official guidelines for quality control of nuclear medicine instrumentation. 


\section{EDUCATION}

A national course on the quality control of nuclear medicine instrumentation was organized and established. Its main objective is to educate and train the persons responsible for carrying out these tasks in each nuclear medicine department. The course was registered at the National College of Health and takes place every 3 years. Forty hours of theoretic and practical sessions are planned, including 20 hours of lectures and 20 hours of laboratory activities. Two pilot courses were delivered in 2002 and 2005, with more than 45 participants (medical physicists and technologists) from the whole country in attendance. The next course is scheduled for the first week of December 2008.

\section{PHANTOM BANK}

A database was created of phantoms and accessories available within Cuba for the quality control of nuclear medicine instrumentation. First, all nuclear medicine departments described and gave technical information on their phantoms and radiation sources and formally agreed to share their resources. A methodology for resource sharing was promoted and administered by the CCEEM. A Web site (www.eqmed.sld.cu/phbank/nmphincu.htm) was created to house the database, which includes clinical phantoms (anthropomorphic torso phantom, cardiac inserts, dynamic cardiac phantom, and others), total-performance phantoms (Carlsson and Jaszczak), calibrated flood and point radiation sources, and linearity and contrast/resolution phantoms. If a nuclear medicine service needs to perform a quality control test using a specific phantom, the service first searches the Web site to find which department has the specific phantom and then asks, in writing, to borrow it. A copy of the letter requesting the loan is sent to the CCEEM, which coordinates the exchange and return dates. The care and transportation of the phan- toms are the responsibility of the department requesting the loan.

\section{AUDIT SERVICE}

An audit service licensed and registered by the Centro Nacional de Seguridad Nuclear (National Center of Nuclear Security) was organized and created. The role of the audit service is to annually assess the state of the instrumentation in all nuclear medicine departments within the country and to evaluate compliance with the quality control programs. More than 10 inspections have been performed by the CCEEM to date and have been useful in improving the quality control programs for nuclear medicine instrumentation. The first inspections indicated that few of the evaluated services had well-established quality control programs, that many of the quality control tests were not performed frequently enough, that some of the measured parameters were not properly registered in the logbook, and that 2 nuclear services were without medical physicists. The findings were recorded in a confidential technical report, which was delivered to the hospital authorities and the chief of the nuclear medicine department. If deficiencies existed, the department was given a year to solve them.

\section{PERFORMANCE EVALUATION}

Finally, measurements were performed in situ in all the national nuclear medicine services to evaluate the current state of gamma-cameras and SPECT systems. The selected tests and procedures were based on the nuclear medicine instrumentation quality control program previously established. The 5 gamma-cameras and 5 SPECT systems in the country were evaluated for uniformity, spatial resolution, sensitivity, energy resolution, linearity, tomographic uniformity, center of rotation, tomographic resolution, and total performance. Nuclear medicine services and equipment were codified to maintain anonymity. Table 1 summarizes the

TABLE 1

Measurements Performed for the Evaluated Instruments

\begin{tabular}{|c|c|c|c|c|c|c|c|c|c|c|}
\hline \multirow[b]{2}{*}{ Device } & \multicolumn{10}{|c|}{ Parameter } \\
\hline & $\begin{array}{c}\text { Intrinsic } \\
\text { uniformity }\end{array}$ & $\begin{array}{c}\text { System } \\
\text { uniformity }\end{array}$ & $\begin{array}{c}\text { System } \\
\text { resolution }\end{array}$ & $\begin{array}{c}\text { Energy } \\
\text { resolution }\end{array}$ & $\begin{array}{c}\text { System } \\
\text { sensitivity }\end{array}$ & Linearity & $\begin{array}{c}\text { Tomographic } \\
\text { uniformity }\end{array}$ & $\begin{array}{c}\text { Center } \\
\text { of rotation }\end{array}$ & $\begin{array}{c}\text { Tomographic } \\
\text { resolution }\end{array}$ & $\begin{array}{c}\text { Total } \\
\text { performance }\end{array}$ \\
\hline Planar 1 & + & + & + & - & + & + & - & - & - & - \\
\hline Planar 2 & + & + & + & - & + & + & - & - & - & - \\
\hline Planar 3 & + & + & + & - & + & + & - & - & - & - \\
\hline SPECT 1 & + & + & + & + & + & + & + & + & + & + \\
\hline SPECT 2 & + & + & + & + & + & + & + & + & + & + \\
\hline SPECT 3 & + & + & + & + & + & + & + & + & + & + \\
\hline SPECT 4 & + & + & + & + & + & + & + & + & + & + \\
\hline SPECT 5 & + & + & + & + & + & + & + & + & + & + \\
\hline Planar 4 & + & - & + & - & + & + & - & - & - & - \\
\hline Planar 5 & + & - & + & + & + & + & - & - & - & - \\
\hline
\end{tabular}




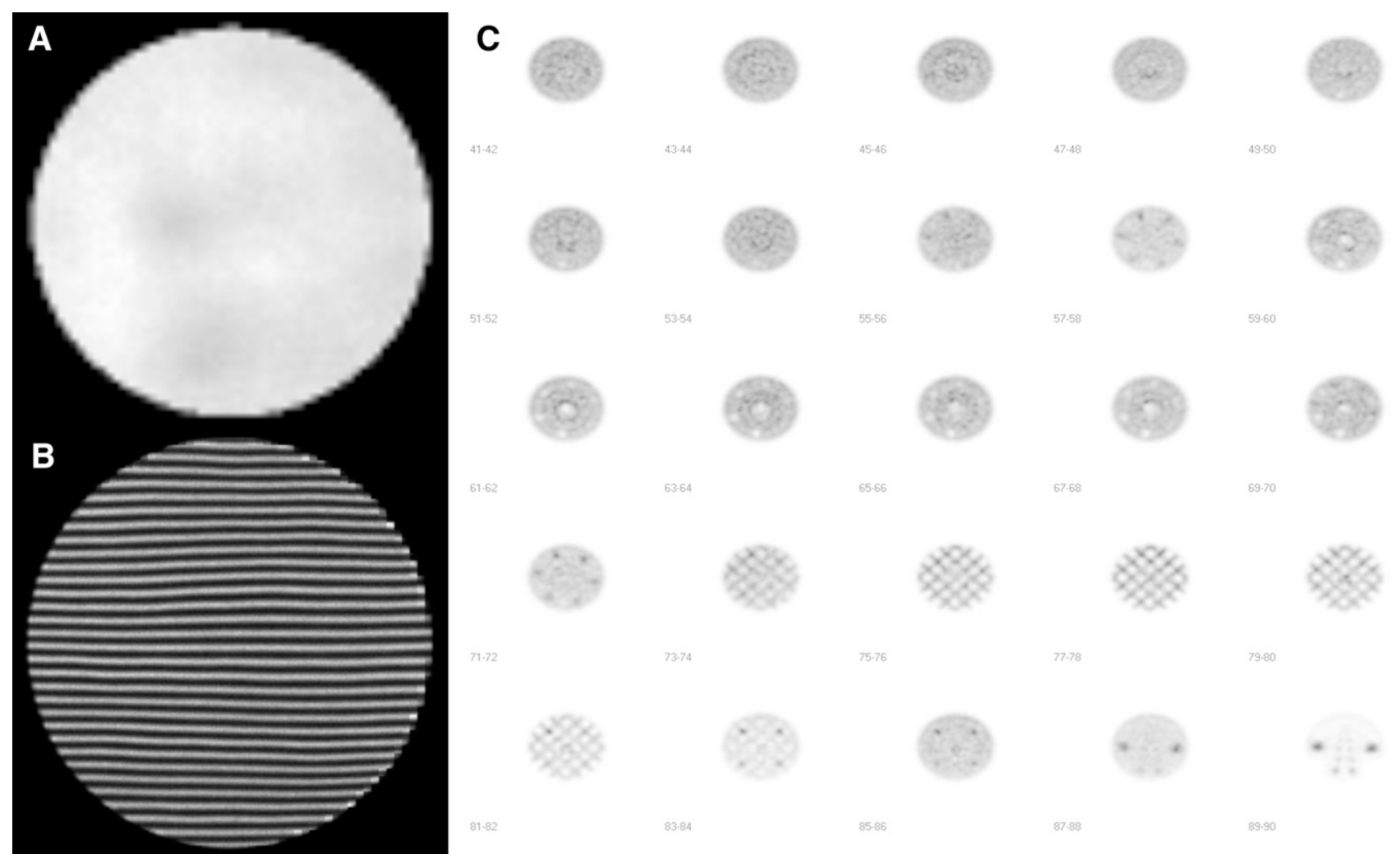

FIGURE 1. Measurements of some parameters: uniformity defects found for SPECT device 2 (A), linearity problems found for planar device $1(B)$, reconstructed slices of different sections of Carlsson phantom obtained with SPECT device 1 (C).

parameters measured on all equipment. In general, the evaluated equipment was in good working order. Some of the detected problems were corrected during the measurement period; in other cases, suggestions were made such that engineering services could fix them. The results were recorded in a formal technical report, which was delivered to the hospital and to the national regulatory authorities. Despite the fact that most of the equipment showed acceptable nonuniformity values (integral and differential uniformities below $5 \%$ for the useful and central fields of view, 2 gamma-cameras (planar device 3 and SPECT device 2) had nonuniformity values over 5\%, which were corrected (Fig. 1A). Alterations in the linearity were also found and corrected on these 2 systems (Fig. 1B). Sensitivity, spatial system resolution, and energy resolution were satisfactory in all evaluated systems.

Once the planar parameters had been tested and corrected if necessary, the tomographic uniformity, center of rotation, and tomographic resolution of the SPECT systems were calculated and evaluated, showing acceptable values in all equipment. Total performance was estimated using a Carlsson phantom filled with a homogeneous solution of ${ }^{99 \mathrm{~m}} \mathrm{Tc}$. Reconstructed slices of the different sections (uniformity, resolution/contrast, linearity, etc.) were evaluated qualitatively and classified as acceptable for all SPECT systems (Fig. 1C). The results of these in situ measurements have been useful in defining priorities for a national upgrading process. Currently, the 2 gamma-cameras that showed the worse results are being replaced.

\section{DISCUSSION}

Application of the quality control program to all nuclear medicine departments in Cuba has contributed to knowledge of the real technical status of the instrumentation installed in these departments, as well as appropriate procedures to correct discovered errors. In addition, the accessories and phantoms available within the country to perform quality control tests have been documented, and procedures have been established for sharing these resources among nuclear medicine services. Also, the required qualifications for personnel responsible for the quality control of nuclear medicine instrumentation have been defined, and these individuals have been trained through national courses approved by the CCEEM. A main result of the project was the development and approval of a national protocol, using international references that were adapted to Cuban national requirements, as well as the instrumentation, accessories, and phantoms available in the country. These, along with the audit service, were implemented and approved by the CCEEM and substantially improved the quality of all nuclear medicine services in the country. It is the first time that Cuba has had a coordinated quality control program for nuclear medicine instrumentation, and the program has made possible the uniform evaluation of all nuclear medicine instrumentation. 
Also, the audit service annually assesses the state of the instrumentation and the qualifications of the personnel. The protocol has been accepted by the medical physics and technologist communities of Cuba, and they are routinely applying it in their nuclear medicine services and sending us their suggestions and comments to improve the protocols. In the future, because of upgrading of older instrumentation, the protocol and courses will need to incorporate quality control procedures for new instrumentation introduced in the country.

We believe that this program can act as a model not only for national nuclear medicine instrumentation programs in other small countries but also for rural programs in larger countries. It may also serve as a model for larger institutions with nuclear medicine instrumentation located and managed at multiple sites such as a large central hospital with several satellite facilities. We believe that many of the challenges that we face in our national program are similar to those at these large, multisite institutions. In these cases, as in ours, a coordinated nuclear medicine instrumentation quality control program that includes uniform quality control protocols, an education program, a resource-sharing program, and periodic auditing will substantially improve the overall quality of nuclear medicine services.

\section{CONCLUSION}

The national program for quality control of nuclear medicine instrumentation was certified and officially estab- lished in 2004 by the Cuban national regulatory authorities (CCEEM) of the Ministry of Health. Similar programs can be organized and established in other countries that lack local regulations for these important activities.

\section{ACKNOWLEDGMENTS}

We thank Marlenin Diaz, Vivian Freixas, Cruz Dumegnico, Joaquin Gonzalez, and the Swedish professor Mats Stenström for their great contribution to data acquisition, the creation and revision of the quality control protocol, and other complementary activities. This work was supported by the Cuban Agency of the Nuclear Energy and Advanced Technologies.

\section{REFERENCES}

1. Quality Control of Nuclear Medicine Instruments. Vienna, Austria: IAEA; 2000.

2. Performance Measurements of Scintillation Cameras. Rosslyn, VA: NEMA; 2001.

3. Hines H, Kayayan R, Colsher J, et al. National Electrical Manufacturers Association recommendations for implementing SPECT instrumentation quality control. J Nucl Med. 2000;41:383-389.

4. Hines H, Kayayan R, Colsher J, et al. Recommendations for implementing SPECT instrumentation quality control: Nuclear Medicine Section-National Electrical Manufacturers Association (NEMA). Eur J Nucl Med. 1999;26: $527-532$.

5. Protocolo Nacional del Control de Calidad en la Instrumentación en Medicina Nuclear. Comisión Nacional. Sociedad Española de Física, Sociedad Española de Medicina Nuclear, Sociedad Española de Protección Radiológica. España, 1999. 